\title{
BMJ Open Youth friendliness in mental health and addiction services: protocol for a scoping review
}

\author{
Lisa D Hawke, ${ }^{1}$ Kristin Cleverley, ${ }^{1,2,3}$ Cara Settipani, ${ }^{1}$ Maureen Rice, ${ }^{4}$ \\ Joanna Henderson ${ }^{1,3}$
}

To cite: Hawke LD, Cleverley K, Settipani C, et al. Youth friendliness in mental health and addiction services: protocol for a scoping review. BMJ Open 2017;7:e017555. doi:10.1136/ bmjopen-2017-017555

- Prepublication history for this paper is available online To view these files please visit the journal online (http://dx.doi. org/10.1136/bmjopen-2017017555).

Received 28 April 2017 Revised 21 July 2017 Accepted 4 August 2017

\section{(a) CrossMark}

${ }^{1}$ Margaret and Wallace McCain Centre for Child, Youth and Family Mental Health, Centre for Addiction and Mental Health, Toronto, Ontario, Canada

${ }^{2}$ Lawrence S. Bloomberg Faculty of Nursing, University of Toronto, Toronto, Ontario, Canada

${ }^{3}$ Department of Psychiatry, University of Toronto, Toronto,

Ontario, Canada

${ }^{4}$ Department of Clinical

Epidemiology and Biostatistics,

McMaster University, Hamilton,

Ontario, Canada

Correspondence to

Dr Joanna Henderson;

joanna.henderson@camh.ca

\section{ABSTRACT}

Introduction To better reach youth in need of mental health and addiction (MHA) services, there is increasing emphasis on making MHA services 'youth friendly.' However, it is unclear what youth friendliness means on a practical level. This scoping review explores (1) how youth friendliness in mental health services is defined in the literature, (2) what characteristics make MHA services youth friendly and (3) how youth friendliness is expected to impact service use by young people.

Methods A search will be conducted of eight electronic bibliographic databases over the last 15 years (20022017) to identify literature on youth friendliness consistent with the modern youth experience. Grey literature will also be searched. The search and literature selection process will include all study designs, as well as nonresearch literature. Two independent raters will determine eligibility based on a review of the titles and abstracts of the identified literature, followed by full text reviews when required. Data will be extracted from the identified literature and then synthesised using qualitative and quantitative approaches. As a final step, we will conduct stakeholder consultations with youth, family members and service provider groups to validate the findings and identify any characteristics of youth friendliness that they deem important that were not reported in the findings. Ethics and dissemination Results will be disseminated through a peer-reviewed publication, as well as international, national and local conference presentations. Agency reports will be developed to present the findings in a user-friendly format, including a youth-friendliness checklist for youth-serving organisations. Research ethics approval has been obtained for the consultation component of this study.

\section{BACKGROUND}

Adolescence is a key period for the development of mental health challenges and mental illness, with some $70 \%$ of mental disorders manifesting before adulthood. ${ }^{1}$ In Canada, it is estimated that one in five youth experience at least one significant mental health or substance use challenge, while suicide is second leading cause of death among youth. ${ }^{2-4}$ Untreated mental health and addictions (MHA) problems in youth come at a

\section{Strengths and limitations of this study}

- This scoping review will provide an enhanced understanding of the definition, characteristics and expected impacts of youth friendliness in mental/ behavioural health and addiction care settings.

- The scoping review results will be strengthened by consultations with youth, family members and community service providers on the definition, characteristics and expected impacts of youth friendliness.

- A limitation of the scoping review methodology is that it does not thoroughly assess the quality of the work included.

- This review will include only English language work with a relatively narrow search focus; the grey literature search will be representative rather than exhaustive.

high cost for the individual and society, yet most youth who would benefit from mental healthcare do not receive treatment. ${ }^{4-6}$

The youth and emerging adult period is a critical age range for development and the establishment of autonomy; however, there are many barriers to MHA treatment during this period. While the United Nations defines 'youth' as those between the ages of 15 and $24,{ }^{7}$ definitions vary in terms of exact age given contextual, cultural and lifestyle issues. Barriers to service utilisation in this age range include inadequate screening and identification of those in need, system fragmentation, access barriers and a lack of evidence-based treatment for this developmental stage..$^{8-12}$ Additional treatment barriers include stigma, a lack of awareness of mental health challenges and services and a preference for self-management over clinical treatment. ${ }^{1314}$ Together, these numerous barriers make MHA services less accessible and appropriate for youth.

There is increasing emphasis on making mental healthcare for youth 'youth friendly.' The World Health Organization (WHO) has 
underlined the importance of youth friendliness in health services as a whole around the world and undertaken efforts to increase the youth focus of such services. ${ }^{15} 16$ Their recommendations include ensuring that services are effective, safe, community-based, confidential and accessible. It is expected that by making services more youth friendly, youth will be more likely to use those services, engage more fully with them and be satisfied with the services they receive. However, this framework was developed for health services as a whole, and with a global perspective, including issues such as sexual health in developing countries, which may or may not transfer to MHA services. While some literature is available on youth friendliness in MHA services, ${ }^{17} 18$ the definitions and characteristics of youth friendliness specific to the MHA sector are unclear.

In the mental health and addictions sector, what does 'youth friendliness' mean on a practical level? How is youth friendliness defined? What characteristics should an MHA service organisation embody to maximise youth friendliness? What are the necessary characteristics of the organisations, the services, the staff and the environment? How is youth friendliness expected to impact young people's use of MHA services? Without a more fulsome understanding of these issues, it is difficult for youthserving MHA organisations to determine the degree of youth friendliness of their services and identify areas for potential improvement. The lack of a clear framework also impedes research on understanding the effect that MHA youth friendliness has on treatment and service outcomes for youth.

\section{OBJECTIVE}

This scoping review will examine the state of the literature on youth friendliness in MHA services from youth, family member and service provider perspectives consistent with the modern youth experience; the focus will be to establish a definition, identify the characteristics and summarise suggested impacts of youth friendliness in youth MHA services.

\section{METHOD}

The methodology for this scoping review will reflect established guidelines. ${ }^{19-22}$ There will be six stages to the methodological approach. We will first define the research question. We will then identify relevant studies through a broad-based search of literature, including both academic and grey literature. We will then select the appropriate studies and extract the data from them, which will be synthesised and reported. Lastly, we will conduct stakeholder consultations with youth, family members and service providers to confirm the findings and identify any missing elements they may suggest, with a view to generating a list of key elements of youth friendliness and identifying directions for future research. For the purposes of this study, 'youth' will refer to young people aged approximately 15-24 based on the United Nations definition, ${ }^{7}$ while allowing flexibility based on the age ranges and definitions of 'youth' used in the literature identified.

\section{Defining the research questions}

This scoping review has three complementary research questions:

- How is youth friendliness in mental health and addiction services defined?

- What characteristics make mental health services youth friendly?

- How does the literature posit that youth friendliness would impact service use by young people?

\section{Identifying relevant literature}

Keywords for the literature search will be developed in collaboration with our youth team members, that is, three Youth Engagement Facilitators who are under the age of 25 and who are staff members on our team, to ensure that the search reflects the concerns raised by youth themselves. Using these keywords, a broad range of literature will be identified, including formal academic literature and grey literature. A search will be conducted of eight electronic bibliographic databases over the last 15 years (2002-2017) to identify literature on youth friendliness consistent with the modern youth experience. We will include in our search all research designs, as well as non-research documents such as guidelines, narrative reviews, policy documents and agency reports. Literature examining youth friendliness in any mental or behavioural health or addiction services care setting will be included. Excluded will be literature focusing solely on physical health services, originating from developing countries or focusing on children or adults outside of the age range reasonably considered to encompass 'youth'.

\section{Search strategy}

Searches, developed by a medical information specialist, will be conducted in the following electronic databases: Medline, EMBASE, PsycINFO (a database of peer-reviewed literature in psychology and related fields), CINAHL (a database of literature in the nursing, allied health, biomedicine and healthcare fields), Applied Social Sciences Index and Abstracts, Campbell Collaboration Library and Cochrane Library. The search will be developed in Medline and adapted to the other databases. The search combines text word and subject heading terms for the main concepts of 'youth friendliness' and adolescent or teen mental or behavioural health. Given the lack of specific subject headings for 'youth friendly' and our intention to focus relatively narrowly on the concept of friendliness, (e.g., we are not interested in the broader concept of 'youth appropriateness'), the search will require that all citations retrieved include either the word 'friendly' or 'welcoming'. In addition to the database search, a grey literature search will be undertaken following the methods outlined in Grey Matters: 
a practical tool for searching health-related grey literature. ${ }^{23}$ Essentially, a focused search will be performed on targeted children/adolescent mental health sites for unpublished materials that meet the inclusion criteria. In addition, Google Advanced Search will be used to undertake a broader grey literature search. References of included studies will be checked for additional sources not found in the search.

\section{Study selection}

Two reviewers will independently review the titles and abstracts of the identified literature to determine eligibility based on an established checklist of eligibility criteria. Conflicts will be resolved by an independent third party. Following the title and abstract review stage, full texts of identified documents will be obtained for more thorough review. The two raters will then review these documents to identify those that meet the inclusion criteria. Any disagreements between the reviewers will be resolved through discussion with a third reviewer to achieve consensus. The review will be conducted in accordance with the Preferred Reporting Items for Systematic Review and Meta-Analysis (PRISMA), including the production of a four-phase PRISMA diagram. ${ }^{24}$ The Covidence online software program for systematic reviews will be used to maximise the efficiency of the review process. ${ }^{25}$

\section{Charting the data}

The data will be extracted from the selected studies using a data extraction form and verified by an independent rater. As per the established methodology for scoping reviews, the quality of the evidence, study or document will not be directly assessed. ${ }^{19}$ However, descriptions of the type and characteristics of selected documents will allow for some discussion of the quality of the literature. Data to be extracted is summarized in table 1 .

\section{Collating, summarising and reporting the results}

The information collected using the data extraction tool will be summarised either qualitatively or quantitatively, as appropriate. This stage will focus on identifying key concepts emerging from the literature. Any quantitative data will be summarised using SPSS V.24. ${ }^{26}$ A table will summarise the articles to describe the current status of work in this area.

\section{Stakeholder consultation}

After summarising the results, we will consult with stakeholder groups to present the results and glean their feedback on them. Our team has standing youth, ${ }^{27}$ family member and community service provider groups whom we regularly consult regarding MHA service provision and research issues; these groups will be leveraged

Table 1. Data to be extracted from identified studies

\begin{tabular}{|c|c|}
\hline & Data to be extracted \\
\hline 1. Descriptive information & $\begin{array}{l}\text { Authors } \\
\text { Title } \\
\text { Reference } \\
\text { Publication type } \\
\text { Publication methodology } \\
\text { Country } \\
\text { Research question addressed } \\
\text { Objectives } \\
\text { Sample size } \\
\text { Participant characteristics } \\
\text { Source of recommendations (youth, emerging adults, family members, service } \\
\text { providers) } \\
\text { Type of mental health and addiction service discussed } \\
\text { Age range of service provision } \\
\text { Age range of youth } \\
\text { Planned outcome of publication (stated next steps) } \\
\text { Use of validated measures }\end{array}$ \\
\hline $\begin{array}{l}\text { 3. Research question } 2 \text { : characteristics of } \\
\text { youth friendliness }\end{array}$ & $\begin{array}{l}\text { Service provider characteristics } \\
\text { Organisational and policy characteristics } \\
\text { Ongoing youth engagement policies } \\
\text { Environment characteristics } \\
\text { Treatment/service characteristics } \\
\text { Other characteristics }\end{array}$ \\
\hline $\begin{array}{l}\text { 4. Research question } 3 \text { : expected impact of } \\
\text { youth friendliness on service utilisation }\end{array}$ & $\begin{array}{l}\text { Expected impact on treatment/service seeking } \\
\text { Expected impact on treatment/service engagement } \\
\text { Expected impact on treatment/service satisfaction } \\
\text { Other expected impacts }\end{array}$ \\
\hline
\end{tabular}


for the consultation phase. Consultation meetings will be held separately with each of the three stakeholder groups, with the goal of approximately 8-10 participants per group. The main findings of the scoping review will be presented to each group; their feedback will then be sought regarding the appropriateness of the findings, their impressions of the accuracy and the identification of any aspects of youth friendliness that they consider to be missing from the literature. This stage will provide the opportunity to validate the findings and round out the results. Participants in the consultation phase will provide informed consent to participate in the study. Research ethics board approval for this phase of the study has been obtained from the Centre for Addiction and Mental Health.

\section{DISCUSSION AND DISSEMINATION}

Results of this scoping review will enable us to define and characterise youth friendliness in MHA services consistent with the current experience of youth. Of particular interest are the characteristics of the service, environment and service providers that make an environment youth friendly. This review will lead to the development of a definition of youth friendliness in the MHA sector and a series of recommendations for youth-serving MHA organisations. It is hoped that through the dissemination of the findings, youth-serving organisations will be spurred to reflect on ways in which they can increase the youth friendliness of their services to maximise their ability to engage young people in a meaningful way, breaking down some of the barriers that prevent youth from accessing and engaging in the MHA services they need.

Results of this review will be disseminated through academic channels in the form of a publication in a peer-reviewed journal in open-access format to ensure access to non-academic partners. International, national and local conference presentations will be planned to further disseminate the results. To maximise dissemination of the findings to youth-serving agencies, a report will be developed presenting the findings in a userfriendly format, including a checklist of youth-friendliness criteria to be considered by service providers. We will also develop a webinar to present our findings to community agencies via Evidence Exchange Network for Mental Health and Addictions (EENet),${ }^{28}$ an online webinar tool that enables sharing of mental health evidence with key stakeholders. Another key initiative in our dissemination strategy will be presentations to our broad network of community partners.

Acknowledgements We thank the YouthCan IMPACT team for their support. We also thank our Youth Engagement Facilitators, Emma McCann, Jackie Relihan and Joshua Miller, for their support in developing this project. KC held the CAMH Chair in Mental Health Nursing Research at the University of Toronto Lawrence S. Bloomberg Faculty of Nursing and Centre for Addiction and Mental Health during preparation of this protocol.
Contributors All the authors equally contributed to the concept and design of the study. LH drafted the protocol. All authors provided critical review and protocol editing through to finalisation. All authors approved the final protocol.

Funding This study is being conducted with the financial support of the Ontario SPOR SUPPORT Unit (OSSU) and also has been made possible through a financial contribution from the Margaret and Wallace McCain Centre for Child, Youth and Family Mental Health at the Centre for Addiction and Mental Health (CAMH). The funders will play no role in the design of the study or the collection, analysis and interpretation of the data.

Competing interests None declared.

Ethics approval Centre for Addiction and Mental Health Research Ethics Board.

Provenance and peer review Not commissioned; externally peer reviewed.

Data sharing statement The search strategies are available on request to the corresponding author.

Open Access This is an Open Access article distributed in accordance with the Creative Commons Attribution Non Commercial (CC BY-NC 4.0) license, which permits others to distribute, remix, adapt, build upon this work non-commercially, and license their derivative works on different terms, provided the original work is properly cited and the use is non-commercial. See: http://creativecommons.org/ licenses/by-nc/4.0/

(c) Article author(s) (or their employer(s) unless otherwise stated in the text of the article) 2017. All rights reserved. No commercial use is permitted unless otherwise expressly granted.

\section{REFERENCES}

1. Kim-Cohen J, Caspi A, Moffitt TE, et al. Prior juvenile diagnoses in adults with mental disorder: developmental follow-back of a prospective-longitudinal cohort. Arch Gen Psychiatry 2003;60:709-17.

2. Bennett K, Rhodes AE, Duda S, et al. A youth suicide prevention plan for Canada: a systematic review of reviews. Can J Psychiatry 2015;60:245-57.

3. Statistics Canada. Table 102-0561. Leading causes of death, total population, by age group and sex, Canada, CANSIM. 2015. http:// www5.statcan.gc.ca/cansim/a26

4. Waddell C, Shepherd C, Schwartz C, et al. Child and youth mental disorders: Prevalence and evidence-based interventions. Vancouver, Canada: Children's Health Policy Centre, Faculty of Health Sciences 2014;15.

5. Costello EJ, He JP, Sampson NA, et al. Services for adolescents with psychiatric disorders: 12-month data from the National Comorbidity Survey-Adolescent. Psychiatr Serv 2014;65:359-66.

6. Office of the Auditor General of Ontario. Annual report of the Auditor General of Ontario. Toronto, Canada, 2008.

7. United Nations Department of Economic and Social Affairs. Definition of youth. http://www.un.org/esa/socdev/documents/youth/factsheets/youth-definition.pdf.

8. Tylee A, Haller DM, Graham T, et al. Youth-friendly primary-care services: how are we doing and what more needs to be done? Lancet 2007;369:1565-73.

9. Wang PS, Berglund P, Olfson M, et al. Failure and delay in initial treatment contact after first onset of mental disorders in the National Comorbidity Survey Replication. Arch Gen Psychiatry 2005;62:603-13.

10. Sterling S, Valkanoff T, Hinman A, et al. Integrating substance use treatment into adolescent health care. Curr Psychiatry Rep 2012;14:453-61.

11. Eisenberg D, Downs MF, Golberstein E, et al. Stigma and help seeking for mental health among college students. Med Care Res Rev 2009;66:522-41.

12. Kozloff N, Cheung AH, Ross LE, et al. Factors influencing service use among homeless youths with co-occurring disorders. Psychiatr Serv 2013;64:925-8.

13. Gulliver A, Griffiths KM, Christensen H. Perceived barriers and facilitators to mental health help-seeking in young people: a systematic review. BMC Psychiatry 2010;10:113.

14. Flisher AJ, Kramer RA, Grosser RC, et al. Correlates of unmet need for mental health services by children and adolescents. Psychol Med 1997;27:1145-54.

15. World Health Organization. Making health services adolescent friendly: developing national quality standards for adolescent friendly health services. Geneva, Switzerland: World Health Organization, 2012. 
16. Mclntyre P. Adolescent friendly health services - an agenda for change. Geneva, Switzerland: World Health Organization, 2002.

17. Hyman SI, Manion I, Davidson S, et al. 'Youth-friendly' characteristics of professionals in mental health settings. Vulnerable Child Youth Stud 2007;2:261-72.

18. Muir K, Powell A, McDermott S. 'They don't treat you like a virus': youth-friendly lessons from the Australian National Youth Mental Health Foundation. Health Soc Care Community 2012;20:181-9.

19. Arksey H, O'Malley L. Scoping studies: towards a methodological framework. Int J Soc Res Methodol 2005;8:19-32.

20. Levac D, Rivard L, Missiuna C. Defining the active ingredients of interactive computer play interventions for children with neuromotor impairments: a scoping review. Res Dev Disabil 2012;33:214-23.

21. Peters M, Godfrey C, Mclnerney P, et al. The Joanna Briggs Institute reviewers' manual 2015: methodology for JBI scoping reviews. Adelaide, SA Australia: The Joanna Briggs Institute, 2015.

22. O'Brien KK, Colquhoun $\mathrm{H}$, Levac D, et al. Advancing scoping study methodology: a web-based survey and consultation of perceptions on terminology, definition and methodological steps. BMC Health Serv Res 2016:16:305.

23. CADTH. Grey matters: a practical tool for searching health-related grey literature. https://www.cadth.ca/resources/finding-evidence/ grey-matters

24. Moher D, Liberati A, Tetzlaff J, et al. Preferred reporting items for systematic reviews and meta-analyses: the PRISMA statement. Ann Intern Med 2009;1519:264.

25. Veritas Health Innovation. Covidence systematic review software. Melbourne, Australia. www.covidence.org.

26. IBM Corp. IBM SPSS Statistics for Windows, Version 24.0. Armonk, NY IBM Corp:2016.

27. Heffernan OS, Herzog TM, Schiralli JE, et al. Implementation of a youth-adult partnership model in youth mental health systems research: challenges and successes. Health Expect 2017.

28. Centre for Addiction and Mental Health. Evidence and exchange network for mental health and addictions, 2016. http://eenet.ca/. 